\title{
EGFR-Mutant Adenocarcinomas That Transform to Small-Cell Lung Cancer and Other Neuroendocrine Carcinomas: Clinical Outcomes
}

Nicolas Marcoux, MD ${ }^{1,12}$; Scott N. Gettinger, MD²; Grainne O'Kane, MD³; Kathryn C. Arbour, MD4 Joel W. Neal, MD5 Hatim Husain, MD; Tracey L. Evans, MD¹3; Julie R. Brahmer, MD ${ }^{8}$; Alona Muzikansky, MA ${ }^{1}$; Philip D. Bonomi, MD ${ }^{9}$; Salvatore del Prete, MD ${ }^{10}$; Anna Wurtz, BS $^{2}$; Anna F. Farago, MD, PhD ${ }^{1}$; Dora Dias-Santagata, PhD; Mari Mino-Kenudson, MD ${ }^{1}$; Karen L. Reckamp, MD ${ }^{11}$; Helena A. Yu, MD Heather A. Wakelee, MD ${ }^{5}$; Frances A. Shepherd, MD³ ${ }^{3}$ Zofia Piotrowska, MD ${ }^{1}$; and Lecia V. Sequist, MD, MPH

PURPOSE Approximately 3\% to $10 \%$ of EGFR (epidermal growth factor receptor) -mutant non-small cell lung cancers (NSCLCS) undergo transformation to small-cell lung cancer (SCLC), but their clinical course is poorly characterized.

METHODS We retrospectively identified patients with EGFR-mutant SCLC and other high-grade neuroendocrine carcinomas seen at our eight institutions. Demographics, disease features, and outcomes were analyzed.

RESULTS We included 67 patients_-38 women and 29 men; EGFR mutations included exon 19 deletion (69\%), L858R (25\%), and other (6\%). At the initial lung cancer diagnosis, 58 patients had NSCLC and nine had de novo SCLC or mixed histology. All but these nine patients received one or more EGFR tyrosine kinase inhibitor before SCLC transformation. Median time to transformation was 17.8 months ( $95 \% \mathrm{Cl}, 14.3$ to 26.2 months). After transformation, both platinum-etoposide and taxanes yielded high response rates, but none of 17 patients who received immunotherapy experienced a response. Median overall survival since diagnosis was 31.5 months (95\% Cl, 24.8 to 41.3 months), whereas median survival since the time of SCLC transformation was 10.9 months (95\% Cl, 8.0 to 13.7 months). Fifty-nine patients had tissue genotyping at first evidence of SCLC. All maintained their founder EGFR mutation, and 15 of 19 with prior EGFR T790M positivity were T790 wild-type at transformation. Other recurrent mutations included TP53, Rb1, and PIK3CA. Re-emergence of NSCLC clones was identified in some cases. CNS metastases were frequent after transformation.

ASSOCIATED CONTENT

Appendix

Author affiliations

and support

information (if

applicable) appear

at the end of this

article.

Accepted on

November 2, 2018

and published at jco.

org on December 14,

2018: Dol https://doi. org/10.1200/JCO.18. 01585

Processed as a Rapid Communication

manuscript.

The current manuscript was offered priority review by Thomas Stinchcombe, MD.

CONCLUSION There is a growing appreciation that EGFR-mutant NSCLCs can undergo SCLC transformation. We demonstrate that this occurs at an average of 17.8 months after diagnosis and cases are often characterized by Rb1, TP53, and PIK3CA mutations. Responses to platinum-etoposide and taxanes are frequent, but checkpoint inhibitors yielded no responses. Additional investigation is needed to better elucidate optimal strategies for this group.

J Clin Oncol 37:278-285. @ 2018 by American Society of Clinical Oncology

\section{INTRODUCTION}

Performing repeat biopsies to study molecular mechanisms of acquired resistance to tyrosine kinase inhibitors (TKIs) in EGFR (epidermal growth factor receptor) -mutant non-small cell lung cancer (NSCLC) has been one of the cornerstones of developing nextgeneration treatment strategies, including the T790Minhibitor osimertinib and combinations of EGFR and MET inhibitors. ${ }^{1,2}$ Repeat biopsy cohorts have also elucidated that approximately $3 \%$ to $10 \%$ of acquired resistance to EGFR TKIs is associated with histologic transformation to small-cell lung cancer (SCLC). ${ }^{3,4}$ Even more rarely, activating EGFR mutation can be identified among de novo SCLCs. ${ }^{5}$
Significant progress has been made in the past few years toward understanding the genetic mechanisms associated with such histologic transformation. For example, Niederst et $a^{6}{ }^{6}$ demonstrated that whereas the founder EGFR mutation is still uniformly found at the DNA level in transformed cancers, expression of the EGFR protein is significantly diminished, thus rendering the transformed tumors unresponsive to EGFR TKIs. Work by Lee et al ${ }^{7}$ suggests that the SCLC clone branches off from the founder clone earlyin some cases even before initial clinical cancer diagnosis - and that cancers prone to transformation may show inactivation of both TP53 and Rb1 at initial NSCLC diagnosis. 
Despite these advances, little is known about the clinical course of patients with EGFR-mutant cancer after SCLC transformation, which leads to uncertainty about appropriate treatments and prognostic implications for clinicians. Here, we describe clinical outcomes from a large retrospective cohort of patients with EGFR-mutant SCLC transformed cancers.

\section{METHODS}

We performed a retrospective chart review of patients with a history of EGFR-mutant SCLC or high-grade neuroendocrine carcinoma-henceforth collectively termed SCLC-who were seen at eight North American cancer centers. Institutional review board approval was obtained independently at each center.

Data that were collected included demographic information, tumor histology and molecular pathology, and clinical treatments and outcomes. Genotyping was performed with a variety of assays, including allele-specific polymerase chain reactions, next-generation sequencing (NGS), and whole-exome sequencing. For some cases with tissue available, older (narrower) genotyping results were expanded and updated using more modern assays for this project. Response and progression assessments were estimated to the best of the investigator's judgment using radiology reports and, when unavailable, physician's notes; formal Response Evaluation Criteria in Solid Tumors (RECIST) measurements or confirmation of response were not obtained. Nevertheless, the general principles that support the RECIST classification, including the magnitude of lesion size variation that defines response and progression, were used to guide the investigators. ${ }^{8}$ In addition, as this cohort was analyzed retrospectively, there was no defined or standard time interval for obtaining response assessments.

Descriptive statistics were developed and time-to-event outcomes were analyzed using the Kaplan-Meier method.

\section{RESULTS}

\section{Baseline Characteristics}

A total of 67 patients with a history of EGFR-mutant SCLC who were treated between 2006 and 2018 were identified at eight cancer centers. The cohort included 38 women and 29 men with a median age at diagnosis of 56 years, a racial makeup of $49 \%$ white and $42 \%$ Asian, with $73 \%$ never smokers (Table 1 ). Fifty-eight patients (87\%) had NSCLC histology at the time of the initial lung cancer diagnosis, predominantly adenocarcinoma, whereas nine patients (13\%) had de novo SCLC or a mixed histology, including SCLC at diagnosis. All patients had EGFR mutations, including 46 (69\%) with exon 19 deletion mutations and 17 (25\%) with L858R. Genotyping platforms used historically at the time of diagnosis for this cohort rarely included the assessment of tumor suppressor genes associated with high-grade neuroendocrine cancers, such as TP53 and $R b 1$, but the prevalence of mutations in these genes was high among the small subset of patients who were tested using NGS (TP53, 100\% [n=7]; Rb1, 50\% [n = 4]; Appendix Table A1, online only).

\section{Pretransformation Course}

The 58 patients with NSCLC at diagnosis received a median of two lines of systemic therapy before SCLC transformation (range, one to six lines), including at least one EGFR TKI in all cases (Table 2). Of note, osimertinib was used as first-line therapy in only one patient. Seventeen patients (29\%) acquired an EGFR T790M mutation and 23 (40\%) received more than one EGFR TKI before transformation. Median total time on EGFR TKIs before transformation was 15.8 months (range, 1.3 to 53.4 months), and median time since diagnosis of advanced NSCLC to SCLC transformation was 17.8 months ( $95 \% \mathrm{Cl}, 14.3$ to 26.2 months; Fig $1 \mathrm{~A}$ ). Nearly all patients $(n=53[93 \%])$ were receiving an EGFR TKI at the time of transformation.

\section{SCLC Characteristics}

At the time of transformation-for the 58 patients who started with NSCLC —or diagnosis - for the nine de novo SCLC cases-histology was reported as classic SCLC in 97\% of cases and as large-cell neuroendocrine carcinoma in the remaining two cases. Additional pathology findings at the time of transformation are summarized in Appendix Table A2 (online only). The founder EGFR mutation was confirmed in all transformed cases that underwent genotyping (Appendix Table A3, online only). Only five SCLC transformed samples harbored an EGFR T790M mutation, including one patient who was known to have had de novo T790M since initial diagnosis, three with prior acquisition of T790M after TKI therapy, and one without any prior documentation of T790M. Mixed NSCLC-SCLC histology was noted in two SCLC cases with T790M. Conversely, at the time of transformation, T790M was not found in 15 (79\%) of 19 patients with prior evidence of the mutation, including in one patient with de novo T790M at initial diagnosis.

The most common mutations identified in SCLC samples were TP53 (38 [79\%] of 48 patients), Rb1 (18 [58\%] of 31 patients), and PIK3CA (14 [27\%] of 52 patients). Frequency of TP53 mutations increased dramatically when considering only samples genotyped by NGS (32 [91\%] of 35 patients), which highlights the low sensitivity of allelespecific polymerase chain reaction assays in detecting non-hot spot mutations in this tumor suppressor (Table 3). No other genes were mutated in a significant portion of cases, with BRCA1 $(n=3)$ being the next most frequently mutated gene in the cohort (Appendix Table A3). Of note, none of the three observed BRCA1 point mutations-Ile1568Met, Ser1294Gly, and Glu686Lys-are thought to be associated with a cancer predisposition syndrome. 
TABLE 1. Demographics of the Study Population

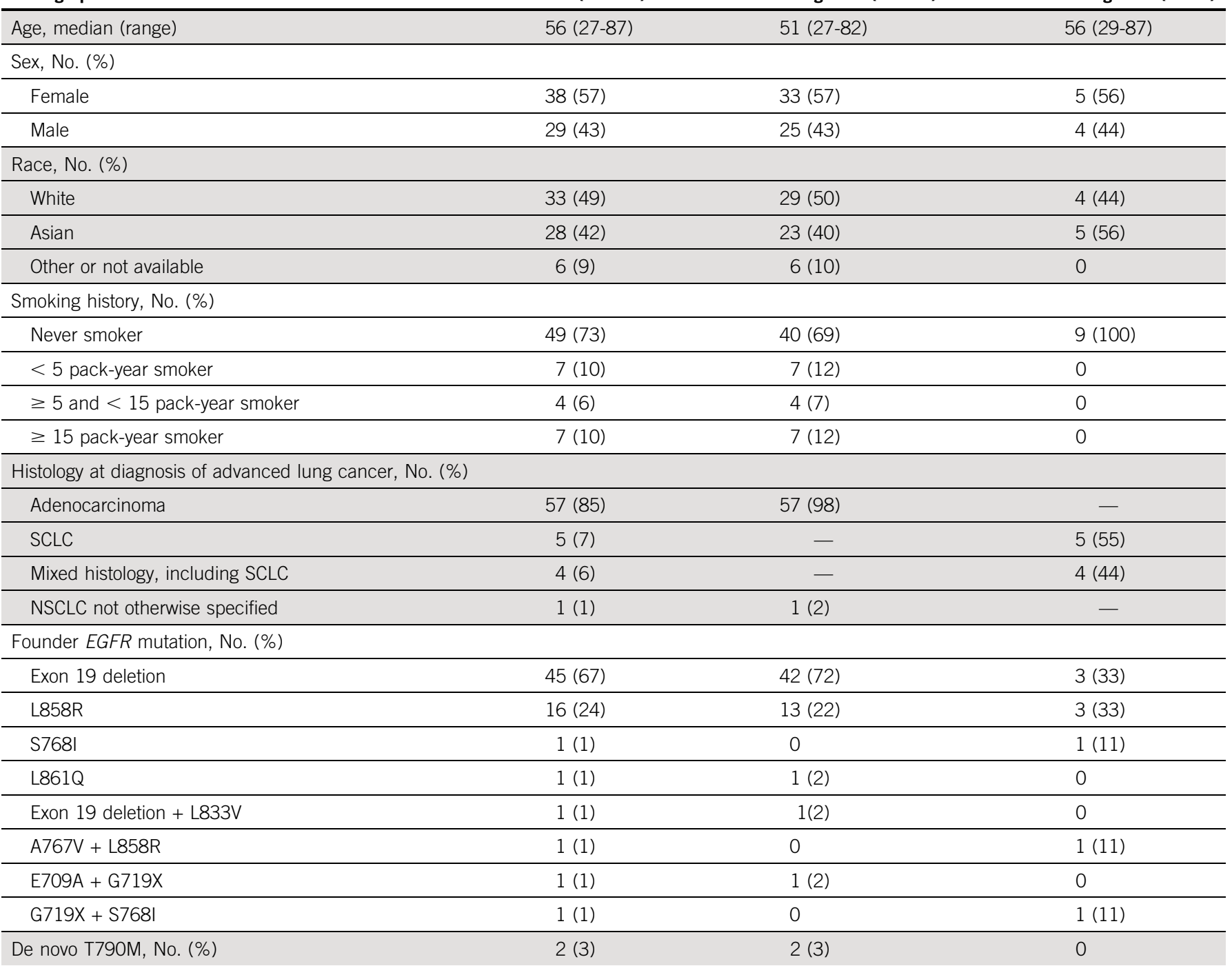

Abbreviations: EGFR, epidermal growth factor receptor; NSCLC, non-small-cell lung cancer; SCLC, small-cell lung cancer.

*SCLC or mixed histology at diagnosis

\section{Post-Transformation Course}

After SCLC transformation-or after diagnosis for patients with de novo SCLC - patients received a median of two lines of systemic treatment (range, zero to six lines; Table 2). As expected, platinum-etoposide was the most commonly used regimen ( $n=53$, including 10 patients who previously received a platinum doublet regimen). Among patients with enough retrospective data for investigators to estimate a response to treatment $(n=46)$, the clinical response rate to platinum-etoposide was $54 \%$. A high clinical response rate to the combination (eight [80\%] of 10 patients) was achieved even in the subset of patients who had previously received platinum chemotherapy for adenocarcinoma. Median estimated progression-free survival on platinum-etoposide was 3.4 months $(95 \% \mathrm{Cl}, 2.4$ to 5.4 months; Fig 1B).
No responses were observed among 17 patients who received a checkpoint inhibitor, either a single-agent programmed death-1 or programmed death-ligand 1 inhibitor $(n=9)$ or as part of the combination ipilimumab-nivolumab regimen $(n=8)$. Indeed, none of the 17 patients even seemed to derive clinical benefit from these therapies, as the longest time to progression was only 9 weeks.

Taxanes were administered to 21 patients, generally late in the course-median of two prior lines of therapy after SCLC transformation-and as single-agent therapy (14 of 21 patients received taxane monotherapy). Among 20 patients with sufficient data to estimate response, clinical response rate to taxane-containing regimens was $50 \%$, including some marked responses (Fig 2). Median estimated progression-free survival on taxanes was 2.7 months (95\% $\mathrm{Cl}, 1.3$ to 3.4 months; Fig 1C). Additional analysis by type of 
TABLE 2. Treatments Received

Therapy Received

No. (\%)

\begin{tabular}{cc}
\hline Received before transformation to SCLC & $\mathrm{n}=58$ \\
\hline EGFR TKI & $58(100)$ \\
\hline Erlotinib & $49(84)$ \\
\hline Afatinib & $13(22)$ \\
\hline Third-generation EGFR TKI & $19(33)$ \\
\hline Osimertinib & $18(31)$ \\
\hline Investigational & $5(9)$ \\
\hline Checkpoint inhibitor & $4(7)$ \\
\hline Cytotoxic chemotherapy & $21(36)$ \\
\hline Platinum-doublet regimens & $20(34)$ \\
\hline Bevacizumab & $9(16)$ \\
\hline
\end{tabular}

Received after SCLC transformation (or after diagnosis for $\quad n=65^{*}$ de novo SCLC)

\begin{tabular}{lc}
\hline Cytotoxic chemotherapy & $63(97)$ \\
\hline Platinum-etoposide & $53(82)$ \\
\hline Other platinum-combination & $7(11)$ \\
\hline Taxane & $21(32)$ \\
\hline Campthotecin (topotecan, irinotecan) & $12(18)$ \\
\hline Temozolamide & $4(6)$ \\
\hline EGFR TKI & $34(52)$ \\
\hline Checkpoint inhibitor & $17(26)$ \\
\hline PD-1 or PD-L1 monotherapy & $9(14)$ \\
\hline Ipilumumab plus nivolumab & $8(12)$
\end{tabular}

NOTE. Only treatments received by at least four patients are listed and patients are listed more than once if they received more than one regimen.

Abbreviations: EGFR, epidermal growth factor receptor; PD-1, programmed death receptor 1; PD-L1, programmed death ligand; SCLC, small-cell lung cancer; TKI, tyrosine kinase inhibitor.

${ }^{*}$ Treatment histories were unavailable for two of the 67 patients in the cohort.

taxane revealed that both paclitaxel and nab-paclitaxel each elicited five responses among seven treated patients (clinical response rate, $71 \%$ ), whereas no responses were observed among six patients who were treated with docetaxel.

Although EGFR TKIs were administered in $52 \%$ of patients after SCLC transformation, they were frequently used in combination with cytotoxic chemotherapy per the treatment-beyond-progression strategy or as maintenance therapy after the conclusion of cytotoxic chemotherapy. ${ }^{9,10}$ Their varied pattern of use limits interpretation of clinical benefit, but a few responses were noted in cases in which concurrently active NSCLC clones were proven or highly suspected. Specifically, although serial biopsies after SCLC diagnosis were not performed in most cases, adenocarcinoma was identified in progressing lesions of at least four patients after SCLC transformation, which suggests more than one active clone concurrently in the same patient. In three of these patients, there seemed to be some degree of clinical benefit gained from EGFR TKI therapy.
As characteristically observed in de novo SCLC, there was a high rate of CNS involvement after SCLC transformation in our cohort. Thirty-eight (64\%) of 59 patients with follow-up radiographic information after SCLC diagnosis experienced progression in the CNS at some point after SCLC diagnosis.

Median follow-up after transformation to SCLC was 8.1 months (range, 0 to 26.9 months) and 45 deaths (67\%) have occurred. Median overall survival since the initial diagnosis of metastatic lung cancer was 31.5 months (95\% $\mathrm{Cl}, 24.8$ to 41.3 months; Fig $1 \mathrm{~A}$ ), and median overall survival since the time of SCLC was 10.9 months $(95 \% \mathrm{Cl}$, 8.0 to 13.7 months; Fig 1D).

\section{DISCUSSION}

To our knowledge, this cohort represents the largest report to date of clinical outcomes for patients with EGFR-mutant lung cancers that either transform to or present initially as SCLC or large-cell neuroendocrine carcinoma. Whereas EGFRmutated de novo SCLC cases are rare, they are likely part of the same biologic continuum as bona fide transformed tumors. Baseline demographic characteristics seem to be relatively similar among our cohort compared with the general population of patients with EGFR-mutant adenocarcinoma whose disease never undergoes such transformation. One characteristic that may distinguish patients with a higher chance of future transformation is the presence of baseline TP53 and/or RB1 mutations, as previously reported by Lee et al. ${ }^{7}$ We observed that SCLC transformation can manifest at any time during the disease course, seen as early as 2 months and as late as 5 years after the diagnosis of metastatic lung cancer, but that the median time to transformation was 17.8 months. After transformation, clinical behavior mimics classic (EGFR wild-type) SCLC on many levels, with frequent but transient responses to platinum-etoposide, frequent CNS metastases, and median overall survival of 10.9 months.

Although the response rate to immune checkpoint inhibitors in pretreated SCLC is relatively modest, complete absence of clinical response in our EGFR cohort, including to anti-programmed death-1/anti-cytotoxic T-cell lymphocyte- 4 combinations, is noteworthy and reminiscent of the poor activity of immunotherapy in more classic EGFR-mutant adenocarcinoma. ${ }^{11-13}$ This suggests that these tumors are biologically more akin to the parental EGFR-mutant adenocarcinoma than to smokingassociated classic SCLC cases. As combination regimens with chemotherapy and immune checkpoint inhibitors have recently demonstrated more promise than singleagent checkpoint inhibitors in both EGFR-mutant adenocarcinoma and de novo SCLC, studying immunotherapy together with chemotherapy could be fruitful in EGFRmutant transformed SCLC. ${ }^{14,15}$

Of equal interest was the relatively high clinical response rate to taxanes observed in EGFR-mutant transformed SCLC, notably to both paclitaxel and nab-paclitaxel $(70 \%$ each), even among heavily pretreated patients. In classic 


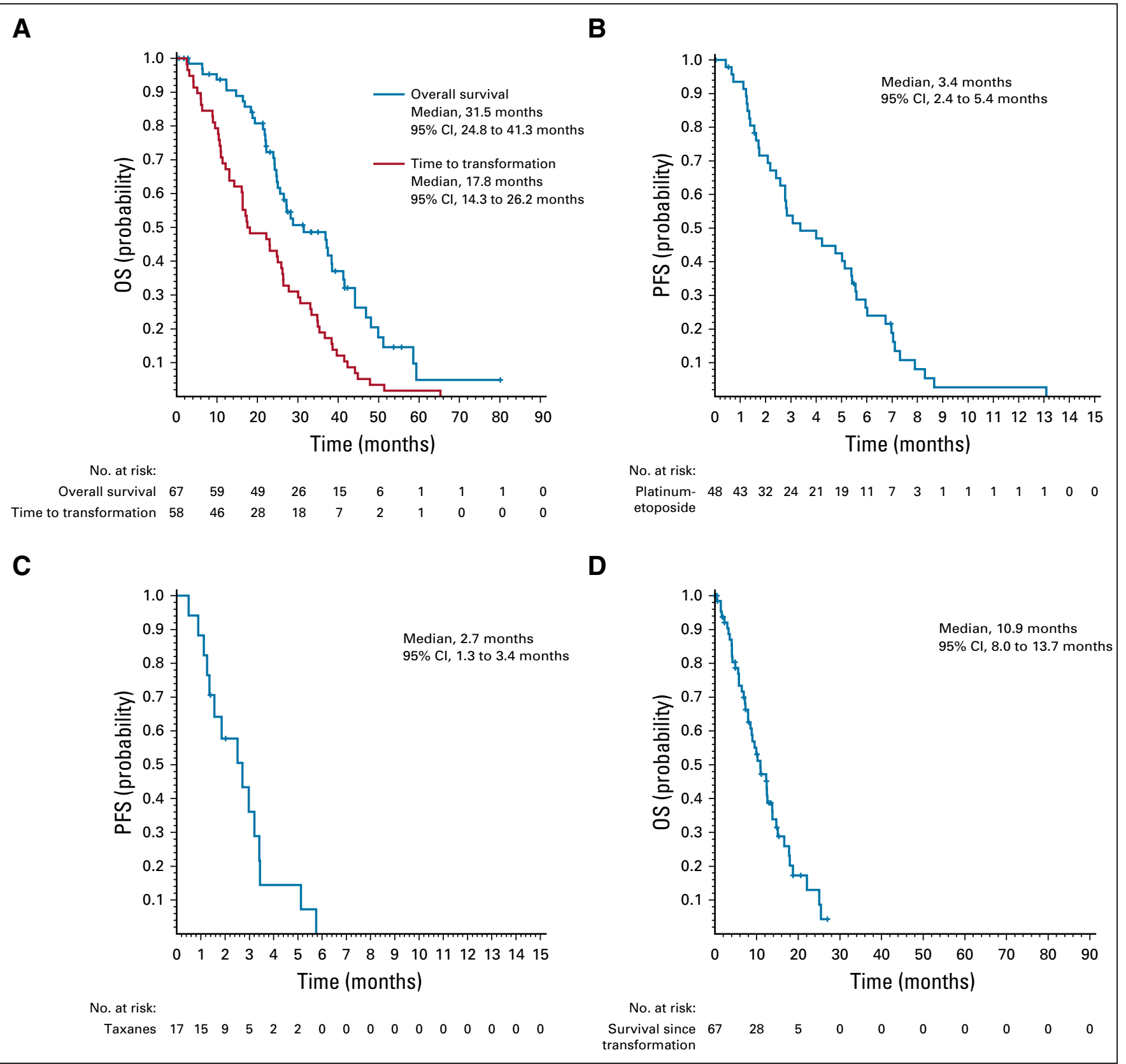

FIG 1. Time to event analyses. (A) Time since diagnosis to transformation to small-cell lung cancer (SCLC) and overall survival (OS) since the time of diagnosis. (B) Progression-free survival (PFS) of SCLC-transformed patients treated with platinum-etoposide. (C) PFS of SCLC-transformed patients treated with taxanes. (D) OS since the time of SCLC transformation.

SCLC, response rate to single-agent taxanes in pretreated patients is only $20 \%$ to $30 \%$, albeit in small studies. ${ }^{16-18}$ It is possible that taxanes are more active in EGFR-mutant transformed SCLC because of residual NSCLC clones that also are responding well to a taxane. Alternatively, EGFRmutant transformed SCLC cells could have a biologic basis for increased sensitivity to taxanes compared with de novo SCLC cases. Nevertheless, despite the small sample size, frequent responses to taxanes were noteworthy and future prospective studies should be considered for this population.
Genotyping was restricted by the historical assays performed and the limited access to additional tissue, although some interesting observations were still possible. Despite few patients tested initially with platforms broad enough to assess TP53 and RB1, high frequency of mutations in these tumor suppressors at diagnosis supports previously reported findings that alterations that affect both genes may strongly predispose to SCLC transformation. ${ }^{7}$ It was rare for SCLC-transformed samples to harbor EGFR T790M, even if it had been previously detected in the patient's prior course, which suggests that the T790M gatekeeper mutation-the 
TABLE 3. Frequency of Common Mutations Within Small-Cell Lung Cancer Cases, by Testing Method

Genotyping Platform

\begin{tabular}{lccr}
\hline All assays & $38 / 48(79)$ & $18 / 31(58)$ & $14 / 52(27)$ \\
\hline Allele-specific PCR & $2 / 8(25)$ & \multicolumn{1}{l}{-} & $3 / 8(38)$ \\
\hline NGS & $32 / 35(91)$ & $15 / 26(58)$ & $11 / 39(28)$ \\
\hline Whole-exome sequencing & $3 / 4(75)$ & $3 / 4(75)$ & $0 / 4(0)$ \\
\hline Unknown & $1 / 1(100)$ & $0 / 1(0)$ & $0 / 1(0)$
\end{tabular}

NOTE. Data are given as No. (\%). One case genotyped only by plasma cell-free DNA analysis is not included in this table (patient 53; Appendix Table A1).

Abbreviations: NGS, next-generation sequencing; PCR, polymerase chain reaction. most common acquired resistance mutation to emerge after first and second-generation EGFR TKIs-tends to reside in a clone that is distinct from the SCLC transformed clone. In other words, the hypothesis of an early branching event between the SCLC clone and the initially predominant NSCLC clonal population, 7 from which EGFR T790Mpositive clones emerge, is consistent with these clinical observations. Mutations that affect TP53, Rb1, and PIK3CA were frequent in the SCLC samples in our cohort, as in other reports. ${ }^{3,4,7}$ Of importance, as a result of the high variability of assays and techniques used in this multicenter cohort, caution should be used when comparing the frequency of mutations with published studies.
Ferrer and colleagues ${ }^{19}$ recently reported on a cohort of 48 EGFR-mutant SCLC transformed cases collected from centers in Europe. Although the cohort had fewer genotyping data available compared with our North American cohort, many similar clinical themes were observed, including a median time since diagnosis to SCLC transformation of 16 months and a median survival after transformation of 9 months.

Our study is limited by its retrospective nature and the fact that treatments and response assessments were not standardized across the cohort. Central review was not performed for pathology slides, nor for radiology scans; however, given the rarity of EGFR-mutant SCLC transformations, the size of the cohort we have collated is significant enough to draw conclusions and inform the treatment of patients in the absence of prospective data. Unfortunately, the current study cannot provide answers to some other relevant questions related to SCLC transformation, such as the impact of first-line use of osimertinib on its frequency or the absolute risk of transformation associated with TP53 and Rb1 mutations at the initial diagnosis of adenocarcinoma. Examining the closely related question of whether there is a signature mutational spectrum of adenocarcinomas that go on to transform to SCLC was limited by access to archival tissue, as many patients from this cohort were initially treated in a community setting and were

\section{A}
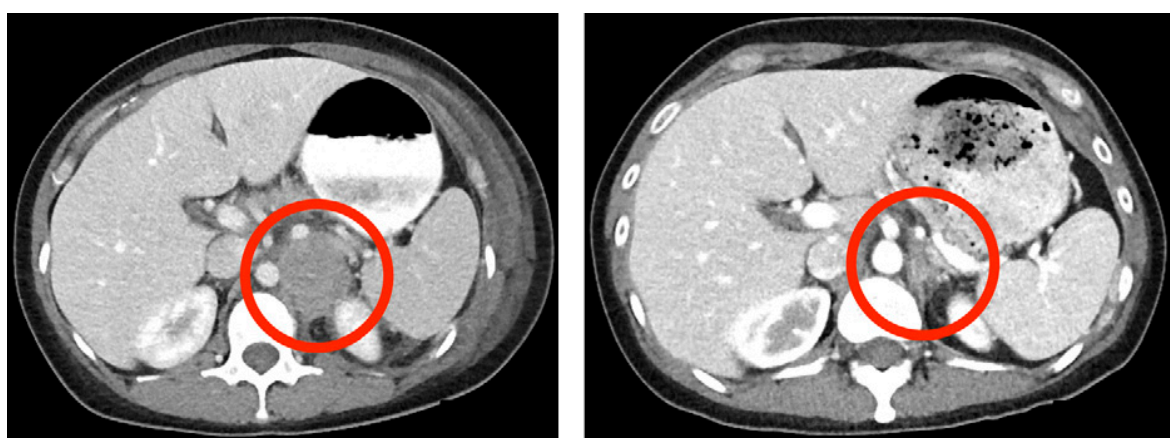

B
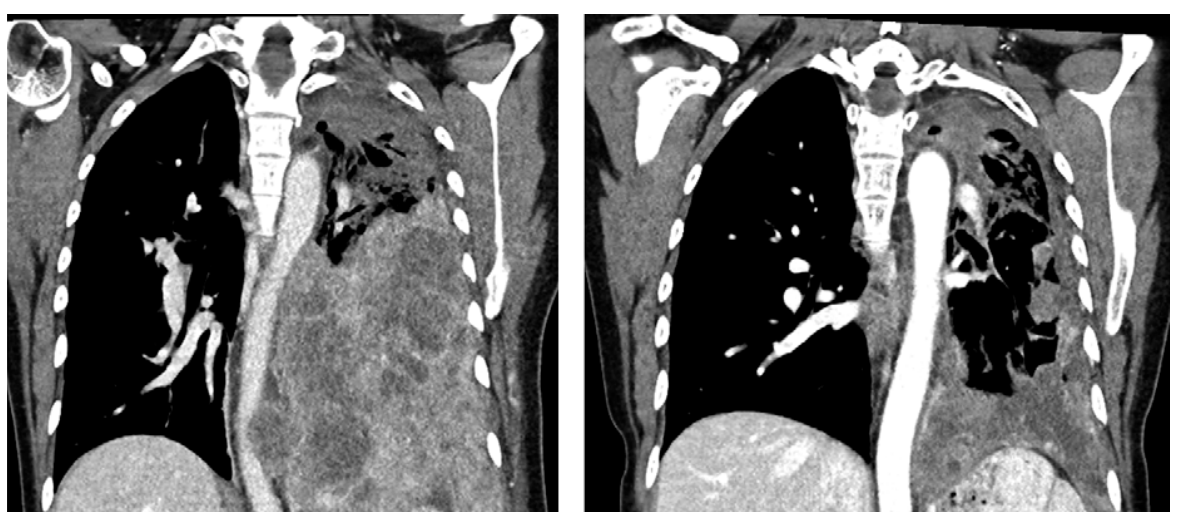

FIG 2. Example of a response to nabpaclitaxel in a SCLC-transformed cancer. (A and B) Significant response of an (A) adrenal metastastasis (red circle) and of (B) extensive thoracic involvement in a patient who had received eight prior lines of therapy for metastatic EGFR (epidermal growth factor receptor) -mutant lung cancer, including singleagent etoposide and a clinical trial of a BCL2/BCL-XL inhibitor since SCLC transformation. 
referred to participating centers later in the course of their disease.

In summary, EGFR-mutant lung cancers that transform to SCLC or that have high-grade neuroendocrine histology at the time of diagnosis exhibit high response rates to platinumetoposide, which should be considered the first-line therapy of choice, and also exhibit high response rates to taxanes. Conversely, these tumors do not respond well to checkpoint inhibitors and the use of these therapies outside of a clinical trial should currently be discouraged. In cases that transformed from initial NSCLC, the founder EGFR mutation was universally maintained and the SCLC and
EGFR T790M-positive clonal subpopulations seemed to be distinct from each other. TP53, Rb1, and PIK3CA mutations are common in SCLC transformations, with the former two also frequent at initial diagnosis among patients whose disease eventually undergoes transformation. Of importance, given the increasing use of cell-free DNA analysis at the time of acquired TKI resistance, our data emphasize the continued role of tissue biopsy at progression for histologic examination, especially in cases in which no genetic resistance mechanism is identified by noninvasive means. Additional investigation and ongoing multicenter collaborations are needed to better elucidate optimal strategies for this group.

\author{
AFFILIATIONS \\ ${ }^{1}$ Massachusetts General Hospital, Boston, MA \\ ${ }^{2}$ Yale Cancer Center, New Haven, CT \\ ${ }^{3}$ Princess Margaret Cancer Centre, Toronto, Ontario, Canada \\ ${ }^{4}$ Memorial Sloan Kettering Cancer Center, New York, NY \\ ${ }^{5}$ Stanford Cancer Institute, Palo Alto, CA \\ ${ }^{6}$ University of California San Diego, La Jolla, CA \\ ${ }^{7}$ Abramson Cancer Center, Philadelphia, PA \\ ${ }^{8}$ Johns Hopkins Sidney Kimmel Comprehensive Cancer Center, \\ Baltimore, MD \\ ${ }^{9}$ Rush University Medical Center, Chicago, IL \\ ${ }^{10}$ Bennett Cancer Center, Stamford CT \\ ${ }^{11}$ City of Hope National Medical Center, Duarte, CA \\ ${ }^{12} \mathrm{CHU}$ de Québec, Quebec City, Quebec, Canada \\ ${ }^{13}$ Lankenau Medical Center, Wynnewood, PA
}

\section{CORRESPONDING AUTHOR}

Lecia V. Sequist, MD, MPH, Massachusetts General Hospital, 32 Fruit St, Yawkey Suite 7B, Boston, MA 02114; e-mail: Ivsequist@partners.org.

\section{PRIOR PRESENTATION}

Presented at the 2018 American Society of Clinical Oncology Annual Meeting, Chicago, IL, June 1-5, 2018, and at the International Association for the Study of Lung Cancer 19th World Conference on Lung Cancer, Toronto, ON, Canada, September 23-26, 2018.

\section{SUPPORT}

Funded by National Institutes of Health Grant No. 2R01CA137008, LungStrong, Targeting a Cure for Lung Cancer, Be A Piece of the Solution, and the Susanne E. Coyne Memorial Fund (L.V.S.), as well as by STOP Cancer Carrie Scott Grant (K.L.R.).

\section{AUTHORS' DISCLOSURES OF POTENTIAL CONFLICTS OF INTEREST} AND DATA AVAILABILITY STATEMENT

Disclosures provided by the authors and data availability statement (if applicable) are available with this article at DOI https://doi.org/ 10.1200/JCO.18.01585

\section{AUTHOR CONTRIBUTIONS}

Conception and design: Nicolas Marcoux, Scott N. Gettinger, Salvatore del Prete, Frances A. Shepherd, Zofia Piotrowska, Lecia V. Sequist

Administrative support: Karen L. Reckamp

Provision of study materials or patients: Kathryn C. Arbour, Tracey L. Evans, Julie R. Brahmer, Philip D. Bonomi, Salvatore del Prete, Anna Wurtz, Anna F. Farago, Dora Dias-Santagata, Karen L. Reckamp, Helena A. Yu, Heather A. Wakelee, Frances A. Shepherd, Lecia V. Sequist Collection and assembly of data: Nicolas Marcoux, Scott N. Gettinger, Grainne O'Kane, Kathryn C. Arbour, Joel W. Neal, Hatim Husain, Tracey L. Evans, Julie R. Brahmer, Philip D. Bonomi, Anna Wurtz, Anna F. Farago, Dora Dias-Santagata, Mari Mino-Kenudson, Karen L. Reckamp, Helena A. Yu, Heather A. Wakelee, Frances A. Shepherd, Zofia Piotrowska, Lecia V. Sequist

Data analysis and interpretation: Nicolas Marcoux, Scott N. Gettinger, Grainne O'Kane, Joel W. Neal, Hatim Husain, Tracey L. Evans, Julie R. Brahmer, Alona Muzikansky, Philip D. Bonomi, Dora Dias-Santagata, Mari Mino-Kenudson, Karen L. Reckamp, Helena A. Yu, Heather A. Wakelee, Frances A. Shepherd, Zofia Piotrowska, Lecia V. Sequist Manuscript writing: All authors

Final approval of manuscript: All authors

Accountable for all aspects of the work: All authors

\section{REFERENCES}

1. Mok TS, Wu YL, Ahn MJ, et al: Osimertinib or platinum-pemetrexed in EGFR T790M-positive lung cancer. N Engl J Med 376:629-640, 2017

2. Ahn M, Han J, Sequist L, et al: TATTON Ph Ib expansion cohort: Osimertinib plus savolitinib for pts with EGFR-mutant MET-amplified NSCLC after progression on prior EGFR-TKI. World Congress on Lung Cancer, Yokohama, Japan, October 15-18, 2017

3. Sequist LV, Waltman BA, Dias-Santagata D, et al: Genotypic and histological evolution of lung cancers acquiring resistance to EGFR inhibitors. Sci Transl Med 3:75ra26, 2011

4. Yu HA, Arcila ME, Rekhtman N, et al: Analysis of tumor specimens at the time of acquired resistance to EGFR-TKI therapy in 155 patients with EGFR-mutant lung cancers. Clin Cancer Res 19:2240-2247, 2013

5. Varghese AM, Zakowski MF, Yu HA, et al: Small-cell lung cancers in patients who never smoked cigarettes. J Thorac Oncol 9:892-896, 2014

6. Niederst MJ, Sequist LV, Poirier JT, et al: RB loss in resistant EGFR mutant lung adenocarcinomas that transform to small-cell lung cancer. Nat Commun 6:6377, 2015

7. Lee JK, Lee J, Kim S, et al: Clonal history and genetic predictors of transformation into small-cell carcinomas from lung adenocarcinomas. J Clin Oncol 35:3065-3074, 2017 
8. Eisenhauer EA, Therasse P, Bogaerts J, et al: New response evaluation criteria in solid tumours: Revised RECIST guideline (version 1.1). Eur J Cancer 45: 228-247, 2009

9. Lo PC, Dahlberg SE, Nishino M, et al: Delay of treatment change after objective progression on first-line erlotinib in epidermal growth factor receptor-mutant lung cancer. Cancer 121:2570-2577, 2015

10. Goldberg SB, Oxnard GR, Digumarthy S, et al: Chemotherapy with erlotinib or chemotherapy alone in advanced non-small cell lung cancer with acquired resistance to EGFR tyrosine kinase inhibitors. Oncologist 18:1214-1220, 2013

11. Antonia SJ, López-Martin JA, Bendell J, et al: Nivolumab alone and nivolumab plus ipilimumab in recurrent small-cell lung cancer (CheckMate 032): A multicentre, open-label, phase 1/2 trial. Lancet Oncol 17:883-895, 2016

12. Lee CK, Man J, Lord S, et al: Clinical and molecular characteristics associated with survival among patients treated with checkpoint inhibitors for advanced nonsmall cell lung carcinoma: A systematic review and meta-analysis. JAMA Oncol 4:210-216, 2018

13. Lisberg A, Cummings A, Goldman JW, et al: A phase II study of pembrolizumab in EGFR-mutant, PD-L1+, tyrosine kinase inhibitor naïve patients with advanced NSCLC. J Thorac Oncol 13:1138-1145, 2018

14. Socinski MA, Jotte RM, Cappuzzo F, et al: Atezolizumab for first-line treatment of metastatic nonsquamous NSCLC. N Engl J Med 378:2288-2301, 2018

15. Liu S, Mansfield A, Szczesna A, et al: IMPower133: Primary PFS, OS and safety in a Ph1/2 study of 1 L atezolizumab + carboplatin + etoposide in extensive-stage SCLC. World Conference on Lung Cancer, Toronto, ON, Canada, September 23-26, 2018

16. Smyth JF, Smith IE, Sessa C, et al: Activity of docetaxel (Taxotere) in small cell lung cancer. Eur J Cancer 30A:1058-1060, 1994

17. Smit EF, Fokkema E, Biesma B, et al: A phase II study of paclitaxel in heavily pretreated patients with small-cell lung cancer. Br J Cancer 77:347-351, 1998

18. Yamamoto N, Tsurutani J, Yoshimura N, et al: Phase Il study of weekly paclitaxel for relapsed and refractory small-cell lung cancer. Anticancer Res 26:777-781, 2006

19. Ferrer L, Giaj Levra M, Brevet M, et al: A brief report of transformation from non-small cell to small cell lung cancer: Molecular and therapeutic characteristics. J Thorac Oncol 10.1016/j.jtho.2018.08.2028 [epub ahead of print on September 11, 2018]

\section{ASCO Immuno-Oncology Program}

Use this eLearning program for an overview of immunobiology along with in-depth topics including treatment, efficacy, monitoring, and the management of immunobiologic agents. Composed of 6 sections that are each designed to becompleted in 15 minutes, users will gain greater competence in the areas of immunobiology, predictive biomarkers, clinical activity, and more. MOC, CME, nursing and pharmacy CE available.

Learn more at university.asco.org/immuno-oncology-program

This course is part of ASCO University Essentials and Advanced Practitioner Certificate Programs.

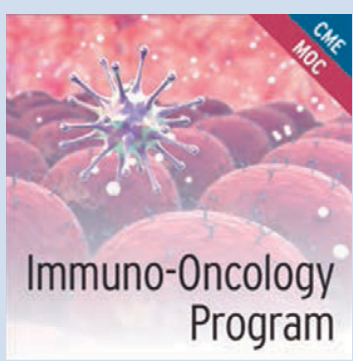




\section{EGFR-Mutant Adenocarcinomas That Transform to Small-Cell Lung Cancer and Other Neuroendocrine Carcinomas: Clinical Outcomes}

The following represents disclosure information provided by authors of this manuscript. All relationships are considered compensated. Relationships are self-held unless noted. I = Immediate Family Member, Inst = My Institution. Relationships may not relate to the subject matter of this manuscript. For more information about ASCO's conflict of interest policy, please refer to www.asco.org/rwc or ascopubs.org/jco/site/ifc.

Nicolas Marcoux

Honoraria: Bristol-Myers Squibb

Scott N. Gettinger

Consulting or Advisory Role: Bristol-Myers Squibb, Alexion Pharmaceuticals, Pfizer

Research Funding: Bristol-Myers Squibb (Inst), Genentech (Inst), Ariad Pharmaceuticals (Inst), Incyte (Inst), Pfizer (Inst)

Travel, Accommodations, Expenses: Merck

\section{Joel W. Neal}

Consulting or Advisory Role: Ariad Pharmaceuticals, Takeda, AstraZeneca, Genentech, Eli Lilly, Exelixis, Loxo, Jounce Therapeutics

Research Funding: Genentech (Inst), Merck (Inst), Novartis (Inst), Boehringe Ingelheim (Inst), Exelixis (Inst), Ariad Pharmaceuticals (Inst), Takeda (Inst), Nektar (Inst)

\section{Hatim Husain}

Consulting or Advisory Role: AstraZeneca, AbbVie, Foundation Medicine Speakers' Bureau: AstraZeneca, Merck, Bristol-Myers Squibb

Research Funding: Pfizer (Inst)

Travel, Accommodations, Expenses: AstraZeneca, Merck, Bristol-Myers

Squibb, Foundation Medicine, AbbVie

\section{Tracey L. Evans}

Honoraria: Genentech, Genentech (I), Merck, AstraZeneca

Consulting or Advisory Role: Genentech, Genentech (I), AstraZeneca

Speakers' Bureau: Genentech (I), Genentech, Merck, AstraZeneca

Travel, Accommodations, Expenses: Genentech, Genentech (I)

Julie R. Brahmer

Consulting or Advisory Role: Bristol-Myers Squibb, Eli Lilly, Celgene, Syndax, Janssen Oncology, Merck, Amgen, Genentech

Research Funding: Bristol-Myers Squibb (Inst), Merck (Inst), AstraZeneca (Inst), Incyte (Inst), Janssen Oncology (Inst)

Travel, Accommodations, Expenses: Bristol-Myers Squibb, Merck

Other Relationship: Bristol-Myers Squibb, Merck

\section{Alona Muzikansky}

Consulting or Advisory Role: Sofregen Medical

Philip D. Bonomi

Honoraria: AstraZeneca, Bristol-Myers Squibb, Biodesix, Merck, Pfizer, Genentech, Trovagene, Eli Lilly

Consulting or Advisory Role: AstraZeneca, Bristol-Myers Squibb, Biodesix, Merck, Pfizer, Genentech, Eli Lilly

Research Funding: AstraZeneca (Inst), Bristol-Myers Squibb (Inst), Corvus Pharmaceuticals (Inst), Five Prime Therapeutics (Inst), Biodesix (Inst), Genentech (Inst), Eli Lilly (Inst), Merck (Inst), Pfizer (Inst)

\section{Anna F. Farago}

Honoraria: Foundation Medicine, DAVAOncology, Clinical Care Options Medical Learning Institute

Consulting or Advisory Role: PharmaMar, Takeda, AbbVie, Loxo, Stemcentrx Genentech, Bayer

Research Funding: PharmaMar (Inst), AbbVie (Inst), AstraZeneca (Inst), BristolMyers Squibb (Inst), Merck (Inst), Loxo (Inst), Ignyta (Inst), Amgen (Inst), Genentech (Inst), Novartis (Inst), Bayer (Inst)

Travel, Accommodations, Expenses: PharmaMar, Stemcentrx, AbbVie, Bayer, Loxo, DAVAOncology, Genentech
Mari Mino-Kenudson

Consulting or Advisory Role: Merrimack Pharmaceuticals, H3 Biomedicine

Karen L. Reckamp

Consulting or Advisory Role: Amgen, Ariad Pharmaceuticals, Astellas Pharma, Euclises, Tesaro, Boehringer Ingelheim, Takeda

Research Funding: Bristol-Myers Squibb (Inst), Pfizer (Inst), Ariad

Pharmaceuticals (Inst), Xcovery (Inst), Adaptimmune (Inst), Genentech (Inst), Boehringer Ingelheim (Inst), AbbVie (Inst), ACEA Biosciences (Inst), Loxo (Inst)

Helena A. Yu

Consulting or Advisory Role: AstraZeneca, Eli Lilly

Research Funding: Clovis Oncology (Inst), AstraZeneca (Inst), Astellas Pharma (Inst), Eli Lilly (Inst), Novartis (Inst), Pfizer (Inst), Daiichi Sankyo (Inst)

Travel, Accommodations, Expenses: Eli Lilly

\section{Heather A. Wakelee}

Honoraria: Novartis, AstraZeneca

Research Funding: Genentech (Inst), Pfizer (Inst), Eli Lilly (Inst), Celgene (Inst),

AstraZeneca (Inst), MedImmune (Inst), Exelixis (Inst), Novartis (Inst), Clovis

Oncology (Inst), Xcovery (Inst), Bristol-Myers Squibb (Inst), Gilead Sciences

(Inst), Pharmacyclics (Inst), ACEA Biosciences (Inst)

Travel, Accommodations, Expenses: AstraZeneca

\section{Frances A. Shepherd}

Stock and Other Ownership Interests: Eli Lilly, AstraZeneca

Honoraria: Eli Lilly, AstraZeneca, Bristol-Myers Squibb, Genentech, Merck Sharp \& Dohme, Merck Serono, Boehringer Ingelheim

Consulting or Advisory Role: Eli Lilly, AstraZeneca, Boehringer Ingelheim, Merck Serono

Research Funding: Eli Lilly (Inst), Pfizer (Inst), Bristol-Myers Squibb (Inst), AstraZeneca (Inst), Medlmmune (Inst), Roche Canada (Inst), Merrimack Pharmaceuticals (Inst)

\section{Zofia Piotrowska}

Consulting or Advisory Role: Boehringer Ingelheim, AstraZeneca, Ariad Pharmaceuticals, Takeda, Superdimension, Guardant Health, Novartis, AbbVie Research Funding: Novartis (Inst), Ariad Pharmaceuticals (Inst), Takeda (Inst), Guardant Health (Inst)

\section{Lecia V. Sequist}

Honoraria: AstraZeneca

Consulting or Advisory Role: AstraZeneca, Genentech, Bristol-Myers Squibb, Pfizer, Merrimack Pharmaceuticals

Research Funding: Boehringer Ingelheim (Inst), Clovis Oncology (Inst)

Genentech (Inst), Merrimack Pharmaceuticals (Inst), Novartis (Inst), AstraZeneca (Inst), Johnson \& Johnson (Inst), Merck (Inst), Pfizer (Inst), Guardant Health (Inst), Incyte (Inst)

No other potential conflicts of interest were reported. 


\section{APPENDIX}

TABLE A1. Frequency of TP53 and Rb1 Mutations in Baseline Tissue, by Testing Method

Genotyping Platform

TP53

RB1

\begin{tabular}{llc}
\hline All assays & $7 / 13$ & $2 / 4$ \\
\hline Allele-specific PCR & $0 / 6$ & - \\
\hline NGS & $7 / 7$ & $2 / 4$
\end{tabular}

NOTE. Data are given as No.

Abbreviations: NGS, next-generation sequencing; PCR, polymerase chain reaction. 
TABLE A2. Summary of Available Pathology Findings of All SCLC Cases

Patient No. Report Available Synaptophysin Chromogranin

\begin{tabular}{llllc}
\hline 1 & Yes & + & + & 90 \\
\hline 2 & Yes & + & & \\
\hline 3 & Yes & + & + & 80 \\
\hline 4 & & & & \\
\hline 5 & Yes & + & + & \\
\hline 6 & Yes & + & + & \\
\hline 7 & Yes & + & + &
\end{tabular}

\begin{tabular}{lllll}
\hline 8 & Yes & & & \\
\hline 9 & Yes & + & + & + \\
\hline 10 & Yes & + & + & + \\
\hline 11 & Yes & + & + & \\
\hline 12 & Yes & + & & \\
\hline 13 & No & & &
\end{tabular}

\begin{tabular}{rrrrrrrr}
\hline 15 & Yes & & + & & & \\
\hline 16 & Yes & + & + & + & $>70$ & - \\
\hline 17 & Yes & + & + & + & 40 & \\
\hline 18 & Yes & + & + & + & 85
\end{tabular}

\begin{tabular}{llllll}
\hline 19 & Yes & + & + & & \\
\hline 20 & No & & & & \\
\hline 21 & Yes & - & - & + & 100 \\
\hline 22 & Yes & + & + & & $>95$
\end{tabular}

hyperchromatic nuclei, and frequent apoptotic bodies; larges areas have nuclear molding and focal areas of necrosis are observed

Small tumor cells with high nuclear/cytoplasmic ratios and speckled chromatin

Majority of cells consistent with small-cell carcinoma

Neuroendocrine features with solid growth, nuclear molding, no obvious mucin production, with nuclear rosettes present and conspicuous apoptotic debris

Consistent with small-cell transformation

Clearly distinct from prior adenocarcinoma

High-grade neoplasm consisting of solid nests of cohesive epithelioid cells with a moderate amount of amphophilic cytoplasm and finely dispersed chromatin without evident nucleoli; there is prominent apoptosis and brisk mitotic activity ( $>20$ mitoses/10 high-power fields); most features consistent with SCLC, but size of tumor cells and amount of cytoplasm greater than that observed in SCLC

Consistent with small-cell carcinoma

-

Consistent with small-cell carcinoma

Consistent with small-cell carcinoma

Large bizarre cells consistent with large-cell neuroendocrine transformation

Small cells with enlarged hyperchromatic nuclei with focal nuclear molding; consistent with small-cell carcinoma

Biphenotypic metastatic carcinoma with predominant cells with high nuclear/cytoplasmic ratios, nuclear molding, and abundant mitoses with focal areas of poorly differentiated adenocarcinoma

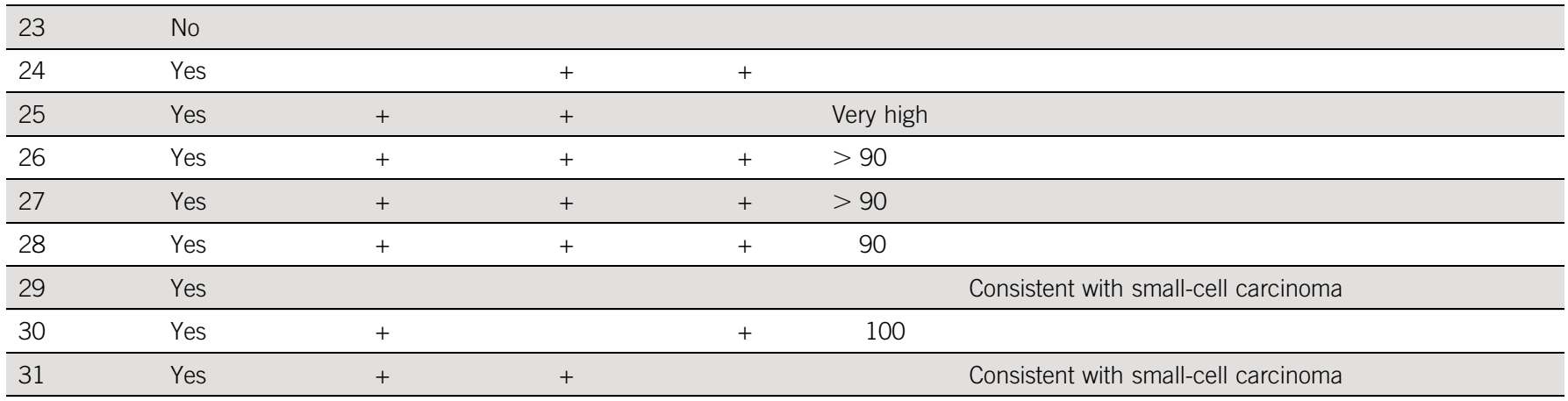

(continued on following page) 
TABLE A2. Summary of Available Pathology Findings of All SCLC Cases (continued)

\begin{tabular}{llllll} 
Patient No. & Report Available & Synaptophysin & Chromogranin & CD56 & Ki-67, \% \\
\hline 32 & Yes & + & + & $>90$
\end{tabular}

\section{Morphologic Description}

Intermediate-sized cells containing scant to focally moderate amounts of cytoplasm, and ovoid to spindled nuclei containing finely dispersed chromatin, without prominent nucleoli; > 10 mitoses/1 high-power field; focal rosette-like structures

\begin{tabular}{|c|c|c|c|c|c|c|}
\hline 33 & Yes & & + & + & 90 & $\begin{array}{l}\text { Tumor cells have round to ovoid pleomorphic } \\
\text { hyperchromatic nuclei, inconspicuous nucleoli, scant/ } \\
\text { minimal faintly eosinophilic cytoplasm, and prominent } \\
\text { nuclear molding; areas of necrosis are present; } \\
\text { apoptotic bodies are readily identified; mitotic activity is } \\
\text { brisk }\end{array}$ \\
\hline 34 & Yes & + & + & + & & $\begin{array}{l}\text { Cells have small amounts of cytoplasm and finely } \\
\text { dispersed nuclear chromatin; mitotic activity is brisk }\end{array}$ \\
\hline 36 & Yes & + & + & + & 90 & $\begin{array}{l}\text { Many relatively small and highly pleomorphic cells with } \\
\text { scanty cytoplasm, inconspicuous nucleoli, and the } \\
\text { stippled chromatin pattern with molding; frequent } \\
\text { mitoses, apoptotic bodies, and necrosis are noted; } \\
\text { occasional cells show larger nuclei, distinct nucleoli, } \\
\text { and a mild amount of cytoplasm }\end{array}$ \\
\hline
\end{tabular}

\begin{tabular}{|c|c|c|c|c|c|c|}
\hline 37 & Yes & + & + & + & 90 & $\begin{array}{l}\text { First component of moderately differentiated } \\
\text { adenocarcinoma demonstrating acinar pattern; second } \\
\text { component of small, round blue cell tumor infiltrating in } \\
\text { solid sheets and possible trabeculae associated with } \\
\text { necrosis; scanty cytoplasm and hyperchromatic nuclei } \\
\text { with high nuclear/cytoplasmic ratio, indistinct nucleoli, } \\
\text { and prominent nuclear molding }\end{array}$ \\
\hline 38 & Yes & + & + & + & 70 & $\begin{array}{l}\text { Solid nests of epithelioid cells with scant cytoplasm, high } \\
\text { nuclear/cytoplasm ratio, and moderate nuclear } \\
\text { pleomorphism }\end{array}$ \\
\hline 39 & Yes & + & + & + & $>90$ & $\begin{array}{l}\text { Sheets of malignant cells containing scant cytoplasm and } \\
\text { hyperchromatic nuclei without prominent nucleoli; up to } \\
8 \text { mitoses/1 high-power field }\end{array}$ \\
\hline
\end{tabular}

\begin{tabular}{llllll}
\hline 40 & Yes & + & + & + & $100 \quad \begin{array}{r}\text { Sheets of malignant small epithelial cells with scant } \\
\text { cytoplasm, hyperchromatic nuclei, and inconspicuous } \\
\text { nucleoli }\end{array}$
\end{tabular}

\begin{tabular}{llll}
\hline 41 & Yes & + & $90 \quad \begin{array}{l}\text { Small neoplastic cells in densely crowded linear and } \\
\text { rounded groups; scant cytoplasm and molded nuclei } \\
\text { with neuroendocrine-type chromatin features; mitotic } \\
\text { figures are readily identified }\end{array}$ \\
\hline
\end{tabular}

42 Yes $\quad+\quad+\quad+\quad+\quad+\quad 90 \quad$ Sheets of tumor cells containing scanting cytoplasm and
hyperchromatic nuclei with high nuclear/cytoplasmic ratio, indistinct nucleoli, and prominent nuclear molding; rare foci of larger cells with slightly more abundant cytoplasm and possible gland formation

\begin{tabular}{|c|c|c|c|c|c|}
\hline 43 & Yes & + & + & & Consistent with small-cell carcinoma \\
\hline 44 & Yes & + & & + & Increased mitosis and occasional single-cell necrosis \\
\hline 45 & Yes & + & - & + & $\begin{array}{l}\text { Cell block shows scattered aggregates and single atypical } \\
\text { cells with elevated nuclear/cytoplasmic ratios and } \\
\text { occasional single filing, irregular nuclear borders, and } \\
\text { smudgy chromatin with conspicuous nucleoli; supports } \\
\text { neuroendocrine differentiation, with features suggestive } \\
\text { of small-cell carcinoma }\end{array}$ \\
\hline
\end{tabular}

(continued on following page) 
TABLE A2. Summary of Available Pathology Findings of All SCLC Cases (continued)

$\begin{array}{lllll}\text { Patient No. Report Available Synaptophysin Chromogranin CD56 Ki-67, \% } & \text { C }\end{array}$

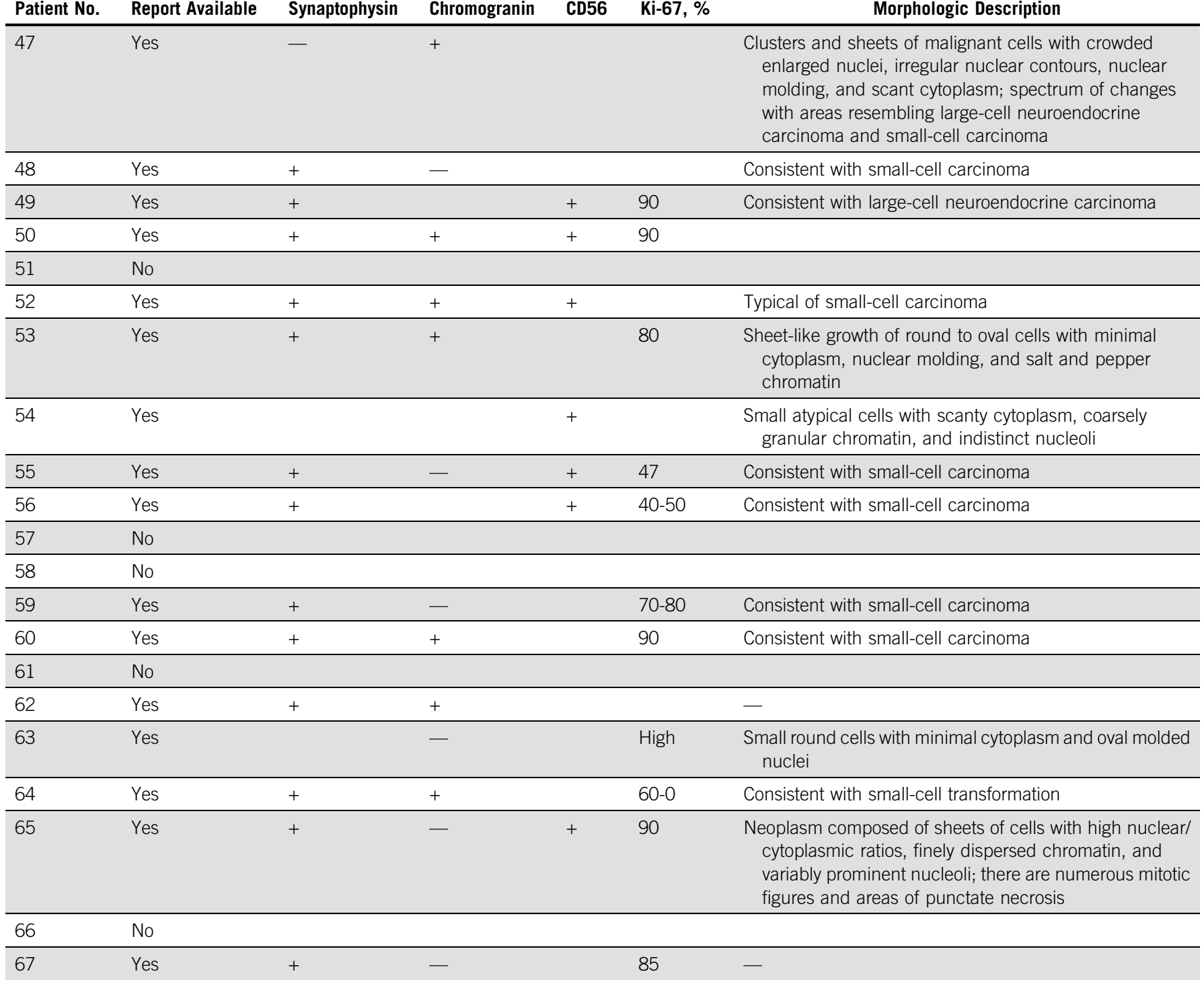

Abbreviation: SCLC, small-cell lung cancer. 
TABLE A3. Genetic Findings of All SCLC Cases

Patient No.

\begin{tabular}{l}
\hline 1 \\
\hline 2
\end{tabular}

7

\begin{tabular}{l}
\hline \\
\hline \\
\hline \\
\hline \\
\hline 8 \\
\hline 9 \\
\hline 10
\end{tabular}

9

10

11

7

12

13
Histology at Diagnosis

Adenocarcinoma

Adenocarcinoma

Adenocarcinoma

Adenocarcinoma

Adenocarcinoma

Adenocarcinoma

Adenocarcinoma

Adenocarcinoma

Adenocarcinoma

Adenocarcinoma

Adenocarcinoma

Adenocarcinoma

Adenocarcinoma

\section{Mutation Reported}

EGFR exon 19 del

EGFR exon 19 del

AKT1 Glu40Lys

TP53 Gly154Val

RB1 Leu343SerfsTer3

PIK3CA Glu542_545GludelinsLyslleThrLys

EGFR exon 19 del

$R B 1$ splice donor variant

NF1 Ile2746Met

TP53 Arg333ValfsTer12

EGFR Leu858Arg

DDR2 Met690Val

TP53 Val197Gly

EGFR exon 19 del

TP53 His193Arg

PIK3CA Glu545Lys

MYC Lys340Arg

EGFR exon 19 del

TP53 Arg306Ter

SMAD4 Cys363Phe

ATM His231Arg

$R B 1$ splice region/intronic variant

EGFR exon 19 del

TP53 Val173Leu

PIK3CA Glu545Lys

PIK3CA Gly726Leu

HER3 Gly337Ala

Biallelic Rb1 loss

FBXW7 Leu8Phe

EGFR Leu858Arg

EGFR exon 19 del

EGFR Leu858Arg

PIK3CA His1047Arg

EGFR Leu858Arg

PIK3CA Glu545Lys

EGFR exon 19 del

TP53 Arg273Leu

EGFR exon 19 del

RB1 Arg320Ter

$F B X W 7$ splice region variant

BRCA1 Glu686Lys

TP53 c.375G $>$ C

PIK3CA Gly545Lys

(continued on following page) 
TABLE A3. Genetic Findings of All SCLC Cases (continued)

\section{Patient No.}

\begin{tabular}{ll}
\hline 14 & Adenocarcinoma \\
\hline 15 & Adenocarcinoma \\
\hline & \\
\hline 16 & SCLC \\
\hline
\end{tabular}

17

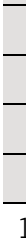

19
SCLC

Adenocarcinoma

Adenocarcinoma

Mixed NSCLC and SCLC

\section{Adenocarcinoma}

Adenocarcinoma

21

\begin{tabular}{l} 
Adenocarcinoma \\
Adenocarcinoma \\
\\
\hline Mixed NSCLC and SCLC \\
\hline Adenocarcinoma \\
\hline Adenocarcinoma \\
\hline Mixed NSCLC and SCLC \\
\hline
\end{tabular}

\section{Mutation Reported}

EGFR exon 19 del

EGFR exon 19 del

EGFR amp

TP53 pPhe109_Arg110del

EGFR exon 19 del

$R B 1 \mathrm{LOH}+$ splice site mutation at exon 22

TP53 LOH + out-of-frame fusion between TP53 exon 1 and ITNL2 exon 8

EGFR Ser768॥le

ABCB11 Ala762Ser

DOCK8 Ala325Thr

NOTCH3 Pro48Leu

RAD51D Ser46Cys

EGFR exon 19 del

TP53 Ile332Asn

EGFR exon 19 del

RB1 Glu352Ter

ATRX Ser519Tyr

TP53 Gln104Ter

TSC2 Ser526Cys

PIK3CA Glu453Lys

PTEN loss

Not genotyped

EGFR Leu858Arg

CIC Ser1058Ter

BRCA1 Ile1568Met

MSH6 His588Tyr

EGFR Leu858Arg

TP53 splice acceptor variant

ALK Pro40Leu

BRCA1 Ser1294Gly

RB1 loss

PTEN loss

MDM2 gain

EGFR Leu858Arg

EGFR exon 19 del

Not genotyped

EGFR Gly719Cys

EGFR Ser768lle

TP53 Cys135Trp

SOX17 (8q11.23) de

LYN (8q12.1) del

PREX2 (8q13.2) del

PRDM14 (8q13.3) del

(continued on following page) 
TABLE A3. Genetic Findings of All SCLC Cases (continued)

Patient No.

Histology at Diagnosis

27

Adenocarcinoma

Adenocarcinoma

Adenocarcinoma

Adenocarcinoma

Mixed NSCLC and SCLC

Adenocarcinoma

32

33

34

35
Adenocarcinoma

Adenocarcinoma

Adenocarcinoma

\section{Mutation Reported}

NOTCH4 Arg1914His

RB1 exon1 splicing variant p.X46_splice

TET1 exon12 Ala1868Val

EGFR exon19 del

EGFR Thr790Met

PIK3CA Glu545Lys

TP53 Arg342Pro

SRC (20q11.23) del

TOP1 (20q12) del

PTPRT (20q13.11) del

IKZF1 Arg511GIn

$R B 1$ exon 12 splicing variant

EGFR Leu858Arg

TP53 Pro152RArgfs*18

RAD21 (8q24.11) amp

MYC (8q24.21) amp

PMAIP1 (18q21.32) amp

ELF3 (1q32.1) amp

PTPRT (20q13.11) amp

TGFBR2 (3p24.1) del

ABL1 Trp423*

CTNNB1 Ser37Phe

CYLD Glu694Leu

HIST1H3H GIn2OProfs*54

MYC Gly295Val

NOTCH2 Glu1025Lys

PIK3C2G Glu1160Asp

RB1 exon 17 splicing variant X500_splice

SMYD3 His206Asp

EGFR exon 19 del

EGFR exon 19 del

EGFR Thr790Met

EGFR exon 19 del

PIK3CA Glu545Lys

EGFR Leu747_Pro753delinsSer

EGFR Gly719Cys

PIK3CA Glu 542 Lys

TP53 Val173Leu

EGFR exon 19 del

EGFR exon 19 del

EGFR Gly719X

EGFR Glu709Ala

TP53 Val157Gly

(continued on following page) 
TABLE A3. Genetic Findings of All SCLC Cases (continued)

Patient No.

\begin{tabular}{l}
36 \\
\hline 37 \\
38
\end{tabular}

36

$$
37
$$

38

39

40

$\frac{40}{41}$

41

42

43

(2)

\section{Histology at Diagnosis}

Adenocarcinoma

Adenocarcinoma
SCLC $†$

Adenocarcinoma

Adenocarcinoma

Adenocarcinoma

Adenocarcinoma

Adenocarcinoma

\section{Mutation Reported}

EGFR exon 19 del

TP53 Val274Phe

Not genotyped

EGFR Ala767Val

EGFR Leu858Arg

EGFR exon 19 del

TP53 Arg282Gly

Not genotyped

EGFR exon 19 del

TP53 c.554_559delinsT

EGFR exon 19 del

TP53 Pro278Ser

EGFR Leu62Arg

EGFR Leu858Arg

MET amp

MAP2K1 amp - equivocal

MYC amp

BCL2L2 amp

CCNE1 amp

FOXP1 Asn505fs*9

NFKBIA amp

NKX2-1 amp

TP53 Phe134Leu

APC Ala2778Ser

ATM Ser310Gly

BRCA2 Val1186lle

CARD11 Ala534Asn + amp

CHD2 Arg1245Pro

DOT1L Leu800Met

PMS2 amp

RAC1 amp

SMAD3 amp

Microsatellite stable

EGFR exon 19 del

KMT2C Leu732Phe

RB1 loss exons 1-23

TP53 Arg65*

ARID1B Met960Thr

ESR1 Gly145Ser

GATA6 Met546Ile

GPR124 Cys1196Tyr

Not genotyped

(continued on following page) 
TABLE A3. Genetic Findings of All SCLC Cases (continued)

\section{Patient No.}

46

47

48

48

49

50

51

52

53

53

(n)

\section{Histology at Diagnosis}

Adenocarcinoma

\section{Mutation Reported}

EGFR exon $19 \mathrm{de}$

TP53 His179Tyr

BRCA2 Thr3033fs*11

RB1 Glu587*

BCL2L2 amp

NFKBIA amp

NKX2-1 amp

ARFRP1 amp

MYC amp

EGFR exon 19 del

TP53 Leu194His

Adenocarcinoma Not genotyped

Adenocarcinoma

EGFR exon 19 del

Adenocarcinoma

Genotyped, but mutations unavailable

Adenocarcinoma

Adenocarcinoma

Adenocarcinoma

Genotyped, but mutations unavailable

Genotyped, but mutations unavailable

(Mutations identified on cfDNA assay)

EGFR exon 19 del

EGFR Glu205Glu

EGFR amp

TP53 Arg175His

PIK3CA Glu545Lys + amp

CCNE1 amp

HER2 amp

RB1 Leu550fs

PIK3CA Val344Gly

APC Ser1345Leu

EGFR Leu858Arg

EGFR exon 19 del

EGFR exon 19 del

EGFR Thr790Met

Genotyped, but mutations unavailable

Genotyped, but mutations unavailable

Adenocarcinoma

Adenocarcinoma

EGFR exon 19 del

PIK3CA Glu545Lys

EGFR exon 19 del

EGFR amp

TP53 Arg248GIn

PIK3CA Glu545Lys

61

Adenocarcinoma

EGFR exon 19 del

PTEN loss exons 2-6

RICTOR amp

FGF10 amp

(continued on following page) 
TABLE A3. Genetic Findings of All SCLC Cases (continued)

Patient No.

Histology at Diagnosis

Mutation Reported

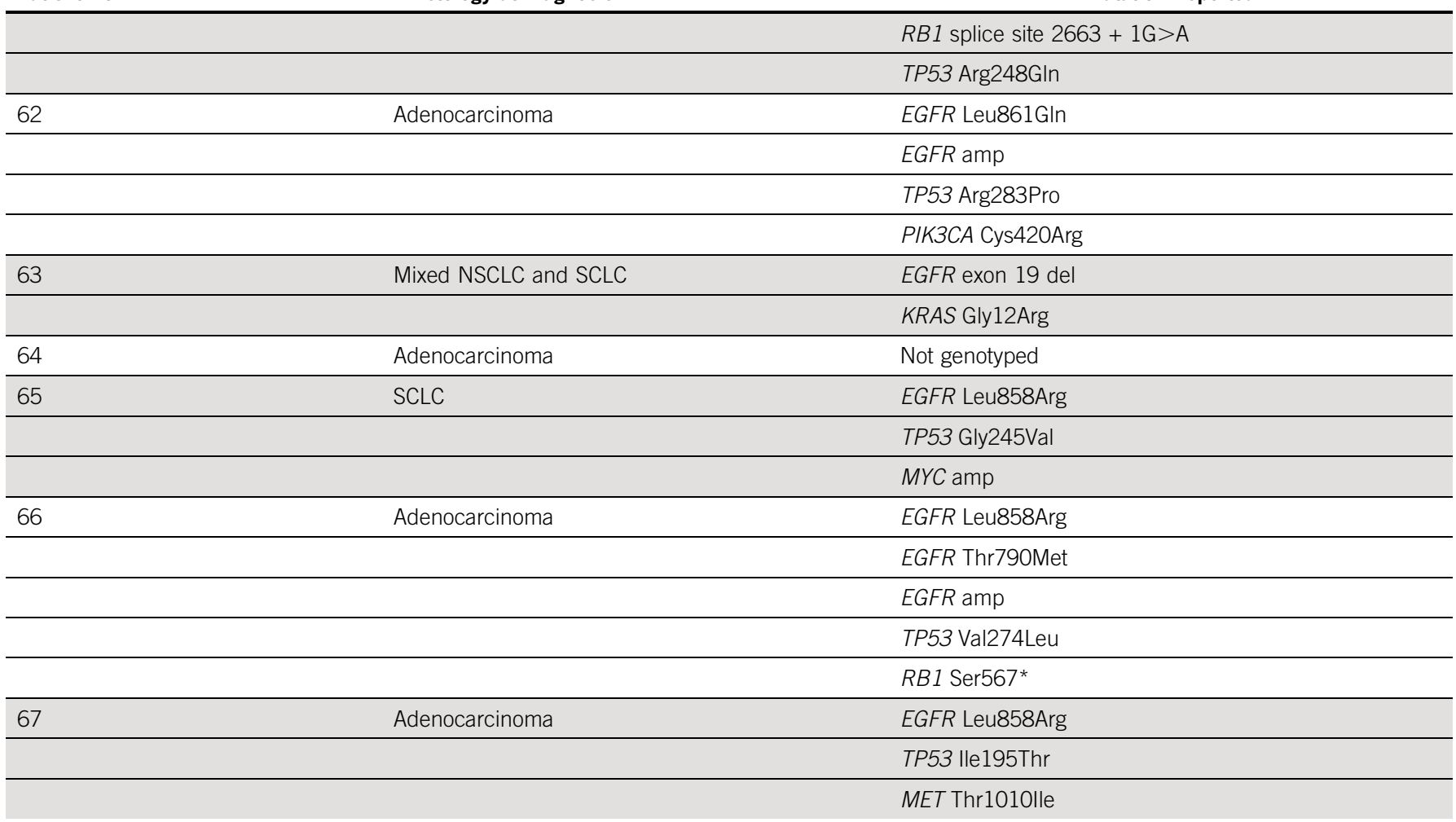

NOTE. Genotyping assay used varies across patients. Similarly, definitions of gene amplification can vary across assays and should be interpreted with caution. Mutations are listed at the protein level unless otherwise indicated.

Abbreviations: EGFR, epidermal growth factor receptor; NSCLC, non-small-cell lung cancer; SCLC, small-cell lung cancer.

†Genotyped after first-line therapy. 